\title{
Thermodynamics of Ethanol + Water + 2-Propanol Mixture at the Range of Temperature 288.15-323.15 K
}

\author{
S. Barros ${ }^{1}$, R.S. Andrade ${ }^{1,2, *}$, and M. Iglesias ${ }^{1}$ \\ ${ }^{1}$ Departamento de Engenharia Química, Universidade Federal da Bahia, Salvador, Brasil \\ ${ }^{2}$ CETENS, Universidade Federal do Recôncavo da Bahia, Feira de Santana, Brasil \\ E-mail: rebeccasa@ufba.br
}

Received 05 April 2017, Revised 13 March 2018, Accepted 15 March 2018

\begin{abstract}
The packing properties of hydroxylic chemicals is very unusual, however these substances find extensive application in food and alcoholic beverage industry. When short alcohols are mixed with water, the entropy of the final system increases far less than we could expected for an ideal solution of randomly interacting molecules. Experimental works on thermodynamics helps to understand how hydrophobic head groups of alcohol molecules in aqueous media cluster together. To this aim, the densities and ultrasonic velocity of the ternary mixtures ethanol + water +2 -propanol at 288.15-323.15 K and atmospheric pressure, have been measured over the whole concentration range, due to the importance of the 2-propanol among the flavor compounds contained into spirit beverages. The experimental data have been analyzed in terms of different theoretical models, an adequate agreement between the experimental and predicted values both in magnitude and sign being obtained, despite the high non-ideal trend. The obtained experimental values indicate varying extent of interstitial accommodation among unlike molecules with strong influence of steric hindrance among aliphatic ends.
\end{abstract}

Keywords: Densities; ultrasonic velocities; derived properties; mixture; estimation procedure.

\section{Introduction}

Knowledge of thermodynamic properties of ethanol, water and the different flavor components in distilled alcoholic beverages is of practical interest for the food industry to obtain a quality final product. Industrial procedures applied to these products are close related on physical magnitudes and their temperature and pressure dependence. Such properties are strongly dependent of hydrogen-bond potency of hydroxyl groups, chain length, isomeric structures, and molecular package. Thermodynamics studies provide the additional advantage of an interesting trend of microscale interaction analysis for understanding macroscale behavior of fluids on mixing. In accordance to that, in the last few years, a considerable effort has been developed in the field of thermodynamic properties, although a great scarce of data is observed in open literature for mixtures enclosed into spirit beverages.

In what is referred to multicomponent mixtures, the scarce of data references is greater, due to the considerable non-ideality, as well as, the complexity of a wide study and the cost/time consuming experimental procedures. Simulation and optimization are not used in a right way in this field, an overestimation of equipment or high energyconsuming conditions being usually applied due to imprecise calculations. In fact, the difficulties for simulation of these processes have been widely commented upon earlier [1-6]. As a continuation of previous works related to flavor components for alcoholic beverages [7-13] we present in this paper the temperature dependence of the density and ultrasonic velocity of the mixture ethanol + water +2 propanol at the range $288.15-323.15 \mathrm{~K}$ and atmospheric pressure, as a function of molar fraction. From the experimental values, the corresponding derived properties (excess molar volumes and change of isentropic compressibility) were computed, a modified temperature dependent Redlich-Kister polynomial being fitted to the results.

Due to the expense of the experimental measurement of such data and current processes design are strongly computer oriented, consideration was also given to how accurate theoretical models work. Despite the importance of computation in chemical processes, the theoretical procedures published in scientific journals are far away of being accurate and of wide application. Our purpose is to discuss the dependence of the excess molar volumes and isentropic compressibility on mixing and other acoustic parameters on the composition and molecular structure, in order to provide a better understanding concerning the factors which contribute to the special behavior in enclosing slight polar molecules into strong hydroxylic environment. The molecular interactions in these systems are complex, and mixing volume and isentropic compressibility data certainly provide valuable information for understanding their molecular trend and packing, as well as being needed for testing of theories of solutions. Different procedures $[12,13]$ were applied for prediction, the obtained results being analyzed, and commented upon. Attending to the deviation of computed data, we arrive at the conclusion that the application of the cubic equations of state with simple mixing rules and the CFT (Collision Factor Theory) model produces acceptable results, despite the high non-ideal trend 
of mixture by hydrogen bond and the wide range of temperature.

\section{Experimental}

All chemical solvents used in the preparation of samples were of Merck quality with richness better than $99.5 \%$. The pure components were stored in sun light protected form and constant humidity and temperature. In order to reduce fraction molar errors, the vapor space into the vessels was minimized during samples preparation. Each vial was weighted with a precision $\left( \pm 210^{-5} \mathrm{~g}\right.$ ) device (GRAM-VXI Serie Analytical Balance), the whole composition range of the ternary mixture being covered. The accuracy in molar fractions was obtained as higher than $\pm 510^{-4}$. The density and ultrasonic velocity were measured with an Anton Paar DSA-5000M device with a precision of $\pm 10-5 \mathrm{~g} \mathrm{~cm}^{-3}$ and \pm $1 \mathrm{~ms}^{-1}$. Calibration of the apparatus was performed periodically, in accordance with technical specifications, using Millipore quality water (resistivity, $18.2 \mathrm{M} \Omega \cdot \mathrm{cm}$ ) and ambient air. Maximum deviation in the calculation of excess molar volumes and changes of isentropic compressibility for these mixtures have been estimated better than $10^{-3} \mathrm{~g} \mathrm{~mol}^{-1}$ and $1 \mathrm{TPa}^{-1}$. The values of the pure components, as well as, open literature data are enclosed into Table 1. More details about techniques and procedure in our laboratory could be obtained from previously published works, as indicated above.

Table 1. Comparison of experimental ultrasonic velocity $\left(u, \mathrm{~ms}^{-1}\right)$ and density $\left(\rho, \mathrm{gcm}^{-3}\right)$ with literature data for chemicals at several temperatures

\begin{tabular}{|c|c|c|c|c|c|}
\hline \multirow[t]{2}{*}{ Component } & \multirow{2}{*}{$\begin{array}{l}\text { Molar } \\
\text { mass }^{\text {a) }}\end{array}$} & \multicolumn{2}{|c|}{$298.15 \mathrm{~K}$} & \multicolumn{2}{|c|}{ Lit. data $(298.15 \mathrm{~K})^{\mathrm{b})}$} \\
\hline & & $\rho$ & $\mathrm{u}$ & $\rho$ & $\mathrm{u}$ \\
\hline \multirow[t]{2}{*}{ Ethanol } & 46.070 & 0.78589 & 1163.64 & 0.78493 & $1143.12^{\mathrm{d})}$ \\
\hline & & & & $0.78730^{\mathrm{c})}$ & $1143.00^{\mathrm{e})}$ \\
\hline \multirow[t]{2}{*}{ Water } & 18.015 & 0.99704 & 1496.89 & 0.99705 & $1496.89^{d)}$ \\
\hline & & & & $0.99712^{\mathrm{c})}$ & $1496.69^{f)}$ \\
\hline \multirow[t]{2}{*}{ 2-Propanol } & 60.096 & 0.78088 & 1139.88 & 0.78098 & $1138.30^{\mathrm{g})}$ \\
\hline & & & & $0.78126^{\mathrm{c})}$ & $1141.00^{\mathrm{h})}$ \\
\hline
\end{tabular}

a) Ref. 14, b) Ref. 15, c) Ref. 16, d) Ref. 17, e) Ref. 18, f) Ref. 19, g) Ref. 20, h) Ref. 21

\section{Correlation of Derived Magnitudes}

The experimental measured data and the corresponding derived magnitudes are presented in Table S1 (supplementary material), the latter being computed from Eq. 1:

$\delta Q=Q-\sum_{i=1}^{N} x_{i} Q_{i}$

In this equation, $\delta Q$ means the variation of a magnitude $\mathrm{Q}\left(\mathrm{V}^{\mathrm{E}}\right.$, excess molar volumes and $\delta \kappa_{\mathrm{S}}$, changes of isentropic compressibilities, calculated by the Laplace-Newton equation from density and ultrasonic velocity experimental data), $\mathrm{Q}_{\mathrm{i}}$ is the pure solvent magnitude (molar volume or isentropic compressibility), $\mathrm{x}_{\mathrm{i}}$ is the mole fraction, and $\mathrm{N}$ is the number of components into the mixtures (Figures 1a and 1b). The data correlation of the corresponding derived magnitude (excess molar volume or changes of isentropic compressibility) was developed using a polynomial expansion like: $\delta Q=\sum_{i=1}^{N} a_{i} x_{i}+\sum_{i=1}^{N} b_{i} x_{i}^{2}+\sum_{i=1}^{N} \sum_{\substack{j=1 \\ j>i}}^{N} c_{i j} x_{i} x_{j}+\sum_{i=1}^{N} \sum_{j=i}^{N} \sum_{k=1}^{N} d_{i j k} x_{i} x_{j} x_{k}$

where $\mathrm{N}$ is the number of components into the mixture and $a_{i}, b_{i}, c_{i j}$ and $d_{i j k}$ are the fitting parameters for the collection of ternary data by the unweighted least squares method, all experimental points weighting equally.

(a)

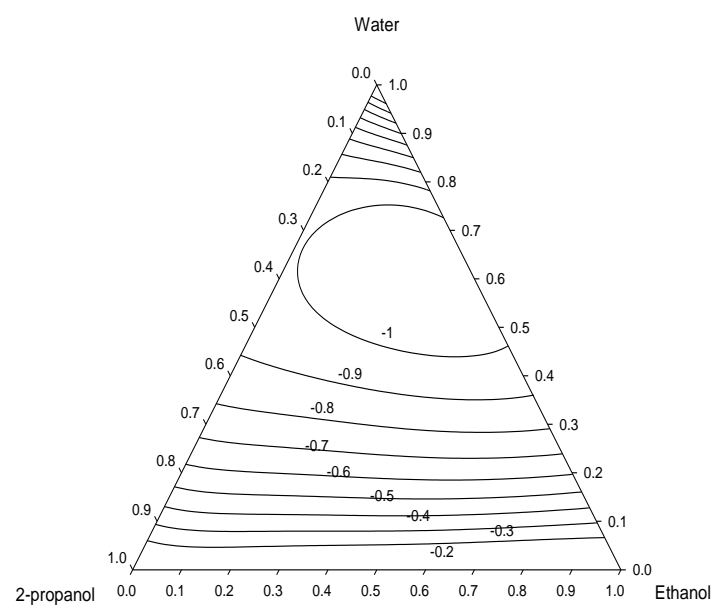

(b)

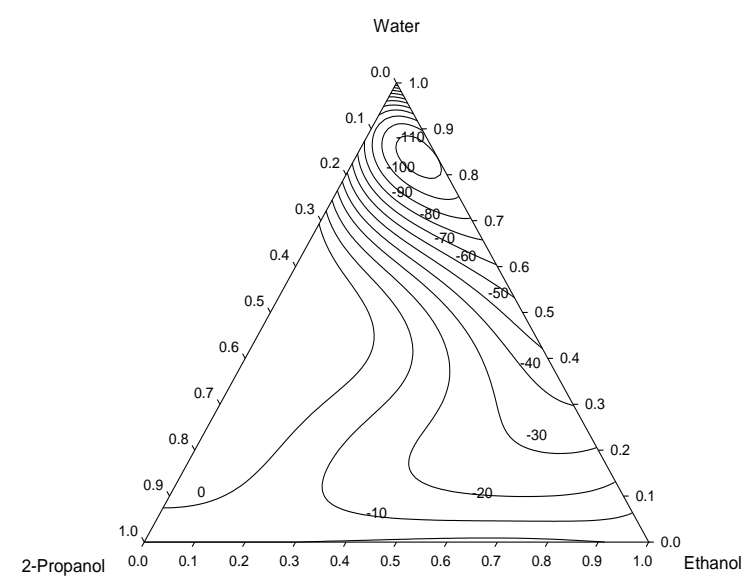

Figure 1. Curves of (a) excess molar volumes $\left(V^{E}, \mathrm{~cm}^{3} \mathrm{~mol}^{-1}\right)$ and $(b)$ changes of isentropic compressibility $\left(\delta \kappa_{\mathcal{S}}, T P a^{-1}\right)$ for Ethanol + Water + 2-Propanol at 298.15 K.

The parameters were computed using a non-linear optimization algorithm due to Marquardt, and were enclosed with their root means square deviations in Table 2 for the temperatures $288.15,298.15$ and $323.15 \mathrm{~K}$.

The root mean square deviations presented were computed using the Eq. 3, where $\mathrm{z}$ is the value of the derived magnitude, and $\mathrm{n}_{\mathrm{DAT}}$ is the number of experimental data:

$\sigma=\left(\frac{\sum_{i=1}^{n_{\mathrm{DAT}}}\left(z_{\mathrm{exp}}-z_{\mathrm{pred}}\right)^{2}}{n_{\mathrm{DAT}}}\right)^{1 / 2}$ 
Table 2. Fitting parameters of Eq. 2 and root mean square deviations $(\sigma)$ for excess molar volumes and changes of isentropic compressibilities for ethanol+water+2-propanol at 288.15, 298.15 and $323.15 \mathrm{~K}$.

\begin{tabular}{r|r|r|r|r|r|r}
\hline & \multicolumn{3}{|c|}{ Excess molar volume } & \multicolumn{3}{c}{ Changes of isentropic compressibilities } \\
\hline & $\mathrm{T}=288.15 \mathrm{~K}$ & $\mathrm{~T}=298.15 \mathrm{~K}$ & $\mathrm{~T}=323.15 \mathrm{~K}$ & $\mathrm{~T}=288.15 \mathrm{~K}$ & $\mathrm{~T}=298.15 \mathrm{~K}$ & $\mathrm{~T}=323.15 \mathrm{~K}$ \\
\hline $\mathrm{a}_{1}$ & -2732.93 & -2732.79 & -2732.78 & -2740.12 & -2737.07 & -2735.33 \\
$\mathrm{a}_{2}$ & -3418.63 & -3418.51 & -3418.50 & -3446.49 & -3439.65 & -3431.51 \\
$\mathrm{a}_{3}$ & 53399.07 & 53399.43 & 53399.45 & 53398.70 & 53407.01 & 53413.45 \\
$\mathrm{~b}_{1}$ & 2765.21 & 2765.09 & 2765.09 & 2762.61 & 2759.83 & 2758.65 \\
$\mathrm{~b}_{2}$ & 2042.07 & 2042.03 & 2042.04 & 1991.04 & 1997.67 & 2008.54 \\
$\mathrm{~b}_{3}$ & 17255.03 & 17254.75 & 17254.76 & 17254.31 & 17249.21 & 17250.24 \\
$\mathrm{c}_{12}$ & 10245.77 & 10246.82 & 10246.92 & 10191.42 & 10217.38 & 10235.87 \\
$\mathrm{c}_{13}$ & -33584.18 & -33581.48 & -33581.44 & -33622.46 & -33571.33 & -33555.40 \\
$\mathrm{c}_{23}$ & -71169.30 & -71167.42 & -71167.30 & -71138.78 & -71088.46 & -71042.31 \\
$\mathrm{~d}_{111}$ & -32.46 & -32.30 & -32.30 & -31.20 & -30.34 & -33.06 \\
$\mathrm{~d}_{112}$ & -4129.00 & -4131.19 & -4131.12 & -4194.57 & -4222.02 & -4206.36 \\
$\mathrm{~d}_{113}$ & -17114.03 & -17118.21 & -17118.30 & -17107.73 & -17193.64 & -17223.31 \\
$\mathrm{~d}_{221}$ & -2723.06 & -2723.98 & -2723.75 & -2812.97 & -2819.68 & -2797.96 \\
$\mathrm{~d}_{222}$ & 1376.11 & 1376.26 & 1376.25 & 1291.48 & 1304.19 & 1317.98 \\
$\mathrm{~d}_{223}$ & 22564.20 & 22557.70 & 22557.96 & 22889.30 & 22814.26 & 22910.97 \\
$\mathrm{~d}_{331}$ & -87734.81 & -87739.40 & -87739.42 & -87684.81 & -87766.24 & -87778.27 \\
$\mathrm{~d}_{332}$ & -49468.91 & -49469.86 & -49469.86 & -49504.66 & -49504.93 & -49481.56 \\
$\mathrm{~d}_{333}$ & -70654.35 & -70654.39 & -70654.35 & -70655.76 & -70659.69 & -70659.75 \\
\hline$\sigma$ & 0.1 & 0.03 & 0.03 & 4.54 & 3.72 & 3.55 \\
\hline
\end{tabular}

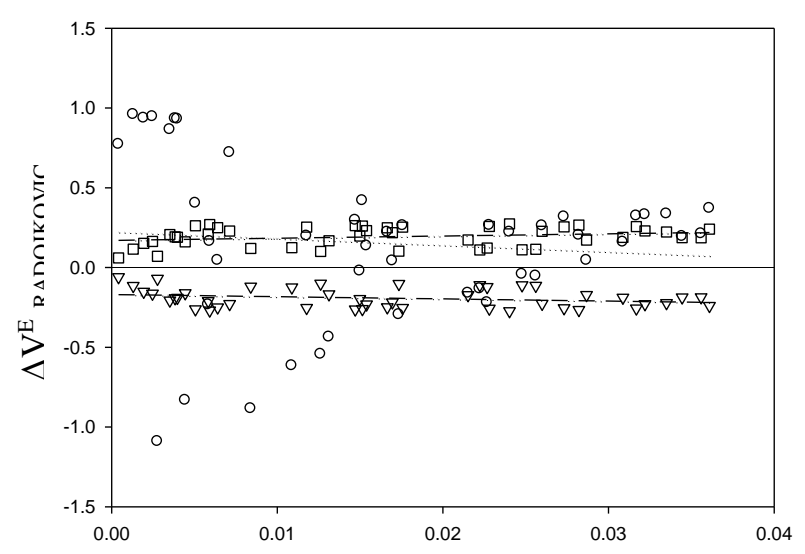

Figure 2. Deviations ( $\triangle V E$, cm3mol-1) of the experimental measured values of excess molar volumes at $298.15 \mathrm{~K}(\mathrm{O}, \cdots$ fitting line) from computed excess molar volumes evaluated by application of the Radojkovic mixing rule [27] (used only binary contributions) on experimental binary data from literature [19, 21]. Limits of deviations of excess molar volumes are enclosed (+25\% (•,- - fitting line), $-25 \%$ $(a,-,-$ fitting line) for comparison.

No previously published data have been found at the studied conditions for this ternary mixture. Only a short collection of density and ultrasonic velocity data for the pure compounds and enclosed binary mixtures was reported at a few temperatures [22-26]. A comparison of these data at $298.15 \mathrm{~K}$ by means of the Radojkovic mixing rule [27] (that only use binary contributions) was made for comparison. Figure 3 shows the deviation between the obtained excess molar volumes from the experimental densities of this work and those binary data from literature using the Radojkovic rule binary. This figure gathers deviations as a function of
$\mathrm{z}=\prod_{\mathrm{i}=1}^{\mathrm{N}} \mathrm{x}_{\mathrm{i}}$ being $\mathrm{x}_{\mathrm{i}}$ the same meaning as explained earlier. This parameter $\mathrm{z}$ points out the difference among pseudo binary compositions (low values of $\mathrm{z}$ ), middle compositions (approximately $\mathrm{z}=0.02$ ) and bulk ternary compositions (high values of $\mathrm{z}$ ). As observed, middle and high $\mathrm{z}$ points gather deviations around $25 \%$ or lower (right side of Figure $3)$. At pseudo binary compositions are gathered large deviations (positive and negative) that suggest poor binary data or low capacity of the mixing rule to express the strong interaction in terms of ternary mixture. Once reviewing the data, it was concluded that the Radojkovic rule has low capacity to express the strong interaction in terms of ternary mixture particularly under the pseudo binary conditions. Curves of the derived magnitudes excess molar volume and changes of isentropic compressibility for the ternary mixture ethanol + water + 2-propanol, have been plotted in Figures 1 and 2, at standard condition. In these figures, a clear contractive tendency is seen for this mixture at low composition of both alcohols, this effect being attenuated towards richer alcohol compositions and higher temperatures. It is worthwhile to point out the large ternary contribution for both magnitudes (the fourth and fifth terms of Eq. 2). As an example, if we compare the values of the derived properties and the corresponding ternary contribution at the same temperature, we may observe that the latter decides the final value and sign of the former. Ternary interactions among dislike molecules are then a key element to quantify the non-ideality of the magnitude and point out the role of each component for diluted mixtures as for alcoholic beverages, where the presence of 2-propanol is in terms of huge dilution (trace flavor compound or so-called congener). 


\section{Acoustic Parameters}

The parameters derived from the experimental measured data were intermolecular free length $\left(L_{f}\right)$, the van der Waals' constant (b), molecular radius (r), geometrical volume $(B)$, molar surface area $(Y)$, available volume $\left(V_{a}\right)$, volume at absolute zero $\left(\mathrm{V}_{0}\right)$, molar sound velocity $\left(R_{S}\right)$, collision factor $(S)$, specific acoustic impedance $(Z)$, and Rao parameter of relative association $\left(R_{A}\right)$, attending to the following set of equations [28-33]:

$L_{f}=\left(\frac{K}{u \cdot \rho^{1 / 2}}\right)$

$b=\left(\frac{M}{\rho}\right)-\left(\frac{R T}{\rho \cdot u^{2}}\right) \cdot\left(\left[1+\frac{M \cdot u^{2}}{3 R T}\right]^{1 / 2}-1\right)$

$r=\left(\frac{3 b}{16 \Pi N}\right)^{1 / 3}$

$B=\left(\frac{4}{3}\right) \Pi r^{3} N$

$Y=\left(36 \Pi N B^{2}\right)^{1 / 3}$

$V_{a}=V\left(1-\left(\frac{u}{u_{\infty}}\right)\right)$

$V_{0}=V-V_{a}$

$R_{S}=\frac{M \cdot u^{1 / 3}}{\rho}$

$S=\frac{u \cdot V}{B \cdot u_{\infty}}$

$Z=u \cdot \rho$

$R_{A}=\left(\frac{\rho_{\operatorname{mix}}}{\rho}\right) \cdot\left(\frac{u}{u_{\text {mix }}}\right)^{1 / 3}$

where $\mathrm{K}$ is a polynomic temperature dependent parameter: $\mathrm{K}=(93.875+0.375 \cdot \mathrm{T}) \cdot 10^{-8}, u_{\infty}$ is taken as $1600 \mathrm{~ms}^{-1}$, and $\mathrm{R}$ and $\Pi$ are common universal constants. These parameters are gathered in Table 3 for the pure compounds at $298.15 \mathrm{~K}$.

(a)

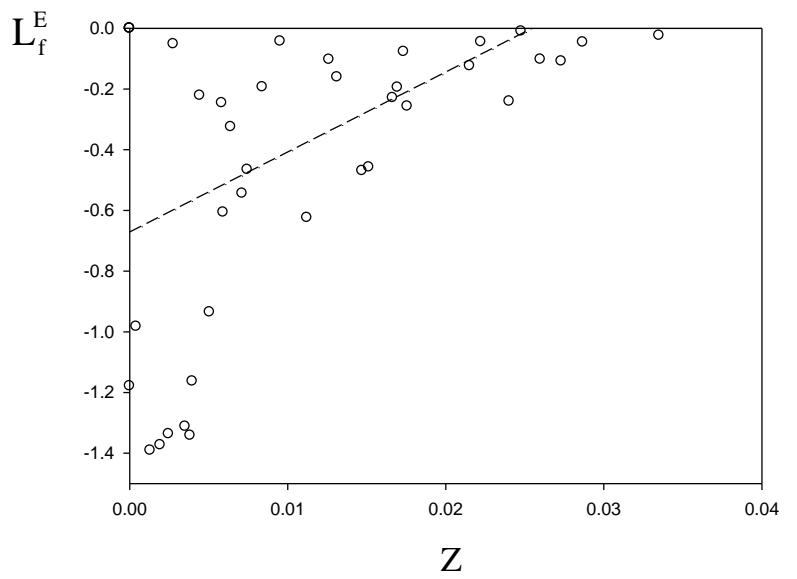

(b)

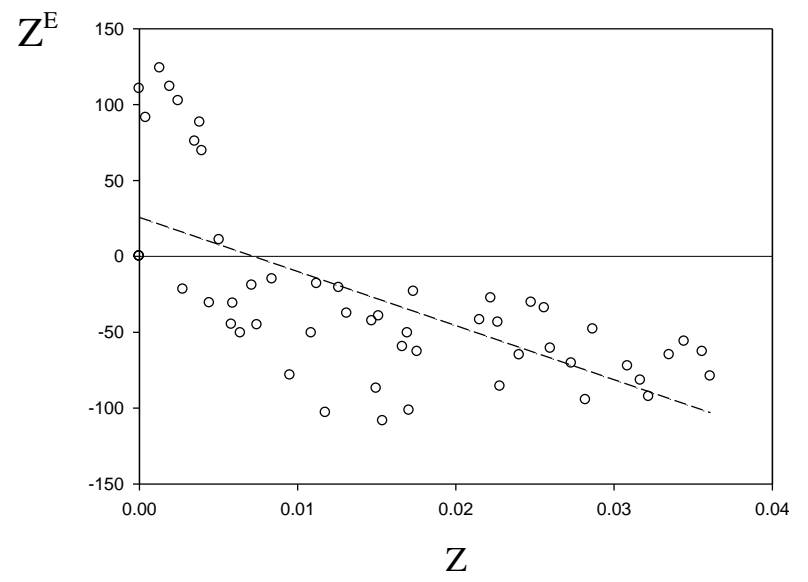

Figure 3. Values of (a) excess intermolecular free length $(\mathrm{O}$, - - fitting line) and (b) excess specific acoustic impedance $(\mathrm{O},-$ - fitting line $)$ for Ethanol + Water + 2-Propanol versus $z$ at $298.15 \mathrm{~K}$.

The variation of the excess magnitudes of $L_{f}$ and $Z$ (by means of Eq. 1) for the ternary mixture at $298.15 \mathrm{~K}$ are shown in the Figures $3 \mathrm{a}$ and $3 \mathrm{~b}$, respectively, showing these magnitudes as a function of $z=\prod_{i=1}^{N} x_{i}$, where $\mathrm{x}_{\mathrm{i}}$ stands for the same meaning as indicated above. In accordance to the contractive trend observed for other ternary mixtures which were studied in our laboratory [8,9], extreme values are gathered at pseudo binaries concentration which reinforce the supposition of the strong distribution of hydroxylic molecules accommodation by the aliphatic end of 2propanol.

\section{Estimation Models}

\subsection{Cubic Equations of State for Excess Molar Volumes}

In the last few years, interest in equations of state for the prediction of thermodynamic properties (excess molar volumes, partial excess and partial molar volumes, phase equilibria, excess molar enthalpies, etc.) has increased. This fact is due to its simplicity as a model, low data requirements (as critical points, acentric factors, etc, Table 4) and versatility in operation conditions. A considerable number of equations of state are available in the literature, most of them being adequate to obtain acceptable results by simple rules if the parameters are obtained from enclosed binary mixtures in the multicomponent system. In this case, the SoaveRedlich-Kwong (SRK) [29] and Peng-Robinson (PR) [30] equations were applied in combination with different mixing rules in the co-pressure and co-volume factors.

For a mixture, at constant $\mathrm{p}$ and $\mathrm{T}$, the excess molar volume is expressed as follows:

$V^{E}=\Delta V=V_{m}-\sum_{i=1}^{n} x_{i} V_{i}=\sum_{i=1}^{n} x_{i}\left(-\left(\frac{\partial p}{\partial n_{i}}\right)_{T, V, n_{j \neq i}}\left(\frac{\partial p}{\partial V}\right)_{T, n_{i}}^{-1}-V_{i}\right)$

where the partial derivatives and the molar volume are obtained from the selected equation of state. A general expression for the applied equations of state is:

$P=\frac{R T}{V-b}-\frac{a}{\left(V+\delta_{1} b\right)\left(V+\delta_{2} b\right)}$ 
Table 3. Acoustic Parameters for the Pure Compounds Enclosed into the Studied Mixture at 298.15 K.

\begin{tabular}{c|c|c|c|c|c}
\hline & $\begin{array}{c}L_{f} \\
(\AA)\end{array}$ & $\begin{array}{c}b \cdot 10^{6} \\
\left(\mathrm{~m}^{3} \mathrm{~mol}^{-1}\right)\end{array}$ & $\begin{array}{c}r \cdot 10^{10} \\
(\mathrm{~m})\end{array}$ & $\begin{array}{c}B \cdot 10^{6} \\
\left(\mathrm{~m}^{3} \mathrm{~mol}^{-1}\right)\end{array}$ & $\begin{array}{c}Y \cdot 10^{-4} \\
\left(\mathrm{~m}^{2} \mathrm{~mol}^{-1}\right)\end{array}$ \\
\hline $\begin{array}{c}\text { Ethanol } \\
\text { Water }\end{array}$ & 19.939 & 53.813 & 1.747 & 13.453 & 23.102 \\
2-Propanol & 13.761 & 16.365 & 1.175 & 4.091 & 5.379 \\
& 20.419 & 71.117 & 1.917 & 17.779 & 27.821 \\
\hline & $\begin{array}{c}\mathrm{V}_{\mathrm{a}} \cdot 10^{6} \\
\left(\mathrm{~m}^{2} \mathrm{~mol}^{-1}\right)\end{array}$ & $\begin{array}{c}\mathrm{R} \\
\left(\mathrm{m}^{3} \mathrm{~mol}^{-1}\right) \cdot\left(\mathrm{m} \mathrm{s}^{-1}\right)^{1 / 3}\end{array}$ & $\mathrm{~S} \cdot 10^{6}$ & $\begin{array}{c}\mathrm{Z} \\
\left(\mathrm{kg} \mathrm{m}^{-2} \cdot \mathrm{s}^{-1}\right)\end{array}$ \\
\hline Ethanol & 13.437 & 616.589 & 3.169 & 914.493 \\
Water & 3.055 & 206.689 & 4.132 & 1492.459 \\
2-Propanol & 17.914 & 803.923 & 3.084 & 890.109 \\
\hline \multicolumn{5}{r|}{}
\end{tabular}

which represents the SRK equation when $\delta_{1}=1, \delta_{2}=0$, and the PR equation when $\delta_{1}=1+\sqrt{2}, \delta_{2}=1-\sqrt{2}$. Three different combining rules were incorporated into these equations, which can be expressed as follows:

$$
\begin{aligned}
& a=\sum_{i=1}^{n} \sum_{j=1}^{n} x_{i} x_{j}\left(1-k_{i j}-l_{i j} X\right)\left(a_{i} a_{j}\right)^{1 / 2} \\
& \mathrm{~b}=\sum_{\mathrm{i}=1}^{\mathrm{n}} \sum_{\mathrm{j}=1}^{\mathrm{n}} \mathrm{x}_{\mathrm{i}} \mathrm{x}_{\mathrm{j}}\left(1-\mathrm{m}_{\mathrm{ij}}\right)\left(\frac{\mathrm{b}_{\mathrm{i}}+\mathrm{b}_{\mathrm{j}}}{2}\right)
\end{aligned}
$$

where $\mathrm{k}_{\mathrm{ij}}, \mathrm{l}_{\mathrm{ij}}$ and $\mathrm{m}_{\mathrm{ij}}$ are fitting parameters to be calculated for each binary mixture, being $\mathrm{l}_{\mathrm{ij}}=\mathrm{m}_{\mathrm{ij}}=0$ for the first mixing rule (R1), $1_{i j}=0$ for the second (R2), and $k_{i j}, 1_{i j}, m_{i j}$ ? 0 and $\mathrm{X}=\left(\mathrm{x}_{\mathrm{i}}-\mathrm{X}_{\mathrm{j}}\right)$ for the last one $(\mathrm{R} 3)$. In every case $\mathrm{k}_{\mathrm{ii}}=\mathrm{l}_{\mathrm{ii}}=\mathrm{m}_{\mathrm{ii}}, \mathrm{k}_{\mathrm{i}}$ $=\mathrm{k}_{\mathrm{ji}}, \mathrm{l}_{\mathrm{ij}}=\mathrm{l}_{\mathrm{ji}}$ and $\mathrm{m}_{\mathrm{ij}}=\mathrm{m}_{\mathrm{ji}}$.

The attraction-cohesion parameter, $\mathrm{a}_{\mathrm{i}}$, contains a generalized function of temperature:

$a_{i}=K_{2} \frac{R^{2} \cdot T_{c i}^{2}}{P_{c i}} \alpha_{i}$

where:

$\alpha_{i}=\left[1+m_{i}\left(1-T_{r i}^{1 / 2}\right)\right]^{2}$

and:

$m_{i}=K_{3}+K_{4} \omega_{i}-K_{5} \omega_{i}^{2}$

in these equations, $\omega_{\mathrm{i}}$ stands for the acentric factor, and $\mathrm{K}_{\mathrm{i}}$ are constant with values $\mathrm{K}_{2}=0.42748, \mathrm{~K}_{3}=0.480, \mathrm{~K}_{4}=1.574$ and $\mathrm{K}_{5}=0.176$ for $\mathrm{SRK}$, and $\mathrm{K}_{2}=0.45724, \mathrm{~K}_{3}=0.37464$, $\mathrm{K}_{4}=1.54226$ and $\mathrm{K}_{5}=0.26992$ for PR.

For every pure substance and both equations, the repulsion-co-volume factor, $b_{i}$, is kept constant at its critical point values, and can be expressed as follows:

$b_{i}=K_{1} \frac{R \cdot T_{c i}}{P_{c i}}$

where $\mathrm{T}_{\mathrm{ci}}$ and $\mathrm{P}_{\mathrm{ci}}$ are the critical temperature and pressure of the component $\mathrm{i}$ in the mixture, and $\mathrm{K}_{1}$ is a constant, which value should be 0.08664 for SRK and 0.0778 for PR.

For pure substances, $T_{c i}, P_{c i}, \omega_{i}$ and $Z_{i}$ values are enclosed in Table 4. For calculating the binary interaction parameters for each mixing rule, the objective function represented by Eq. 23 was minimized.
$O F=\frac{\sum_{i=1}^{n}\left(z_{\exp }-z_{\text {pred }}\right)^{2}}{z_{\exp }}$

Table 4. Open literature critical values for the compounds enclosed into the ternary mixture a)

\begin{tabular}{ccccc}
\hline Compound & $p_{c} / \mathrm{bar}$ & $T_{c} / \mathrm{K}$ & $\mathrm{Z}_{\mathrm{c}}$ & $\omega$ \\
\hline Ethanol & 61.48 & 513.92 & 0.240 & 0.649 \\
Water & 220.64 & 647.14 & 0.229 & 0.344 \\
2-Propanol & 47.62 & 508.30 & 0.248 & 0.665 \\
\hline
\end{tabular}

a) Ref. 14

A Marquardt algorithm was applied to calculate the parameters, in combination with a Newton-Raphson method to solve the equation. From the fitting binary parameters, the excess molar of a higher order multicomponent system can be easily estimated, by applying the same mixing rules, extended for multicomponent cases, and so it was done, obtaining predictions for the ternary excess molar volumes, that were compared with the set of experimental data. After some algebra the objective function $\mathrm{F}$ will be expressed as:

$F=\mathrm{V}_{\text {exp }}^{\mathrm{E}}-\sum_{\mathrm{i}=1}^{\mathrm{n}} \mathrm{x}_{\mathrm{i}}\left(-\frac{\left(\frac{R T}{V-n b}-\frac{n R T}{(V-n b)^{2}} \frac{\partial}{\partial n_{i}}(V-n b)-\frac{1}{\mu} \frac{\partial\left(a n^{2}\right)}{\partial n_{i}}+\frac{a n^{2}}{\mu^{2}} \frac{\partial \mu}{\partial n_{i}}\right)}{-\frac{n R T}{(V-n b)^{2}}+\frac{a n^{2}}{\mu^{2}} \frac{\partial \mu}{\partial n_{i}}}-V_{\mathrm{i}}\right)$

where $\mu=\left\{\left(V+n \delta_{1} b\right)\left(V+n \delta_{2} b\right)\right\}, \mathrm{V}$ is the mixture molar volume, $\mathrm{R}$ is the universal constant of gases, $\mathrm{T}$ the absolute temperature, and $\mathrm{n}$ is the number of moles in the mixture.

In the Figure 4, the accuracy of the different mixing rules could be observed for the corresponding binaries at 298.15 $\mathrm{K}$ and $\mathrm{PR}$ equation, compared with experimental data of different authors [25-27]. The parameters corresponding to Eq. 17 and 18 are enclosed into Table 5.

Despite of the accuracy in correlate the experimental data (proved at Figures $4 \mathrm{a}$ and $4 \mathrm{c}$ ), the prediction of the ternary values quantitatively fails due to two facts: first, the strong interaction among the hydroxylic molecules, secondly, the low contribution of alcohol + alcohol excess values (almost ideal solutions) (see Figure 4b), due to their analogous chemical structure. In this mixture, the intermolecular associations in terms of $\mathrm{H}$-bond by ethanol and water, is in opposition to the 2-propanol, which shows relative steric hindrance and slight polar interactions. Open literature shows as the number of $\mathrm{H}$-bonds per water and per ethanol decrease as the mole fraction of ethanol increases, but this variable is increased when a higher alcohol is also mixed. In 
fact, the entropy of the ternary system increases far less than expected for an ideal solution of ramdomly mixed molecules and the highest contraction occurs at low 2-propanol composition. This wel-known effect has been associated to hydrophobic head groups forming clathrate or ice-like structures in the surrounding water core. As in earlier papers [8-9] our data show that most of the water molecules exist as short strings/clusters associations by $\mathrm{H}$-bond, embedded into a fluid of close packed aliphatic groups, with water clusters bridging neighboring alcohols (hydroxyl groups) by $\mathrm{H}-$ bonds.

This trend is in accordance with the anomalous water + alcohols thermodynamics due to an incomplete molecular level mixing and a residual matrix of the network structure of pure water. As explained previously, larger aliphatic chain into alcohols on mixing deals towards larger entropies and then, low solubility at any cases of high heavy alcohol compositions. High ternary contribution with different character attending to ethanol composition is the cause of the huge values observed for accommodation of the molecules around equimolar pseudo binary ethanol + water system, not surprisingly this package is sensible to the temperature, a diminution of this attraction being noted when temperature rises. As commented above, despite accurate description of binary mixtures by equation of state, slight capability of prediction was obtained, due to the huge ternary contribution with respect the total value of excess molar volume and the low contribution between alcohols.

(a)

$\mathrm{V}^{\mathrm{E}}$

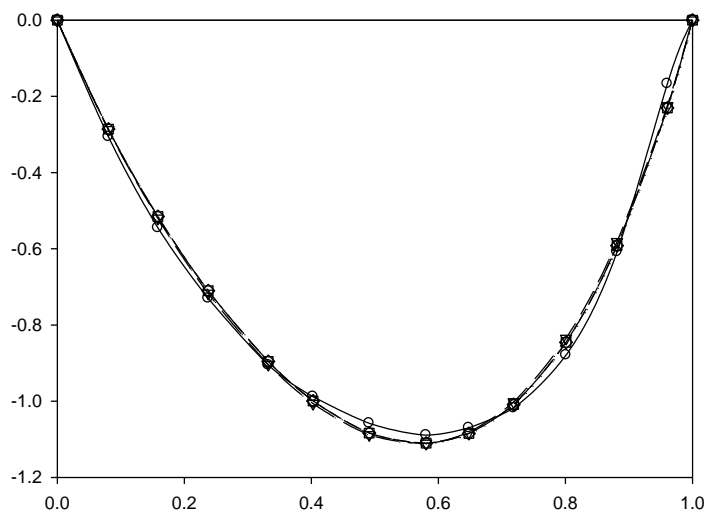

(b)

$\mathrm{V}^{\mathrm{E}}$

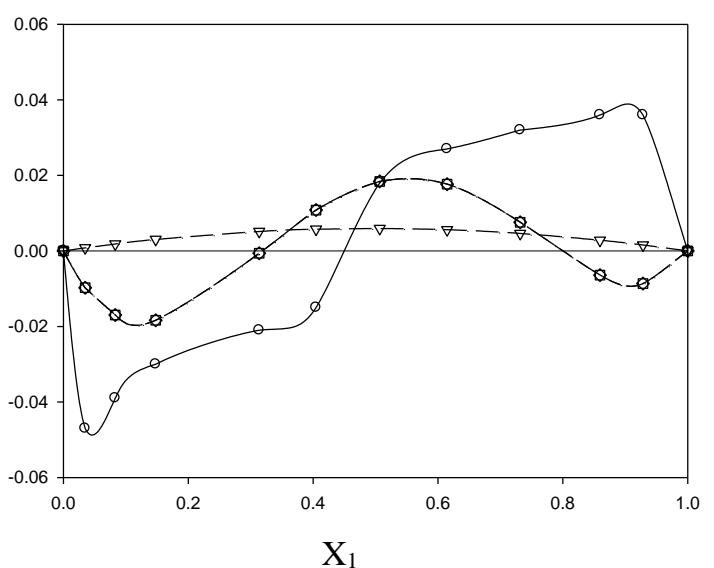

(c)

$\mathrm{V}^{\mathrm{E}}$

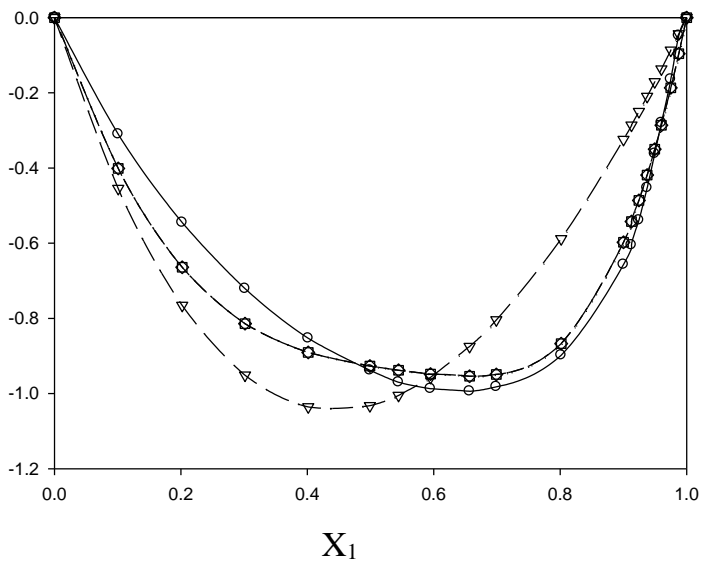

Figure 4. Comparison of the experimental binary excess molar volumes $\left(V^{E}, \mathrm{~cm}^{3} \mathrm{~mol}^{-1}\right)$ from literature [20-22] and Peng-Robinson (PR) equation of state by different mixing rules at $298.15 \mathrm{~K}$, (a) (O) experimental Ethanol + Water mixture; (b) (O) experimental Ethanol + 2-Propanol mixture; (c) (O) experimental Water + 2-Propanol. The figures show the mixing rules applied into the corresponding equation of state as $\left(\nabla,-\_\right)$for mixing rule $R 1,(\square,---)$ for mixing rule $R 2$, and ( $\diamond,-----)$ for mixing rule $R 3$.

Table 5. Computed Binary Interaction Parameters by the Applied Mixing Rules on the Equations of State SRK and PR, and Root Mean Square Deviations of Binary Correlation and for Ternary Prediction (Eq. 3)

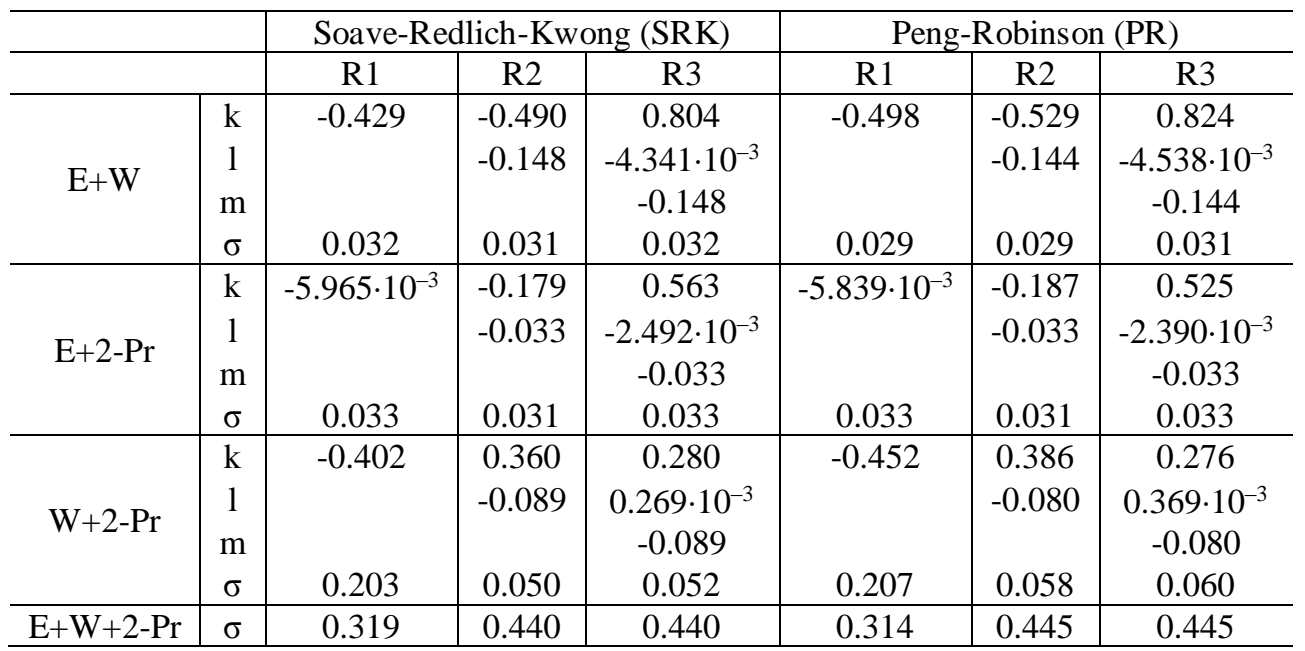


Attending to the obtained results, it is necessary to apply more robust mixing rules (non-quadratic, excess Gibbs energy dependent rules, etc) for a full description of these complex systems (high non ideality, phase split when the aliphatic end rises, etc), poor predicting results being obtained when interactions among three different molecules take place due to this approximation is not considered in the structure of the applied combination mixing rules (clearly symmetric values).

The important non-ideality and the considerable ternary contribution support the idea that similar effects will be observed when the addition of other heavy key components is made on mixtures containing these short polar chemicals. This intense interaction produces high deviations from ideal trend and resulting in a great difficult for modeling and simulation of alcoholic beverages.

\subsection{Collision Factor Theory and Free Length Theory for Isentropic Compressibility.}

Experimental data for the isentropic compressibility of the mixtures were compared with values determined by different mixing procedures. The Collision Factor Theory (CFT), (eq. 25) and Free Length Theory (FLT) (eq. 26) for the isentropic compressibilities were applied [13]:

$\kappa_{S}=\left(\frac{1}{\rho^{3}}\right) \cdot\left(\frac{M}{u_{\infty} \cdot \sum_{i}^{N} x_{i} S_{s} \cdot \sum_{i}^{N} x_{i} B_{s}}\right)^{2}$

$\kappa_{S}=\left(\frac{L_{f}^{2}}{K^{2}}\right)$

The Collision Factor Theory (CFT) is dependent on the collision factors among molecules as a function of temperature into pure solvent or mixture. The pertinent relations in these calculations and its theoretical basis were described in the literature cited above. The collision factors (S) and the characteristic molecular volumes (B) of the pure solvents used in the CFT calculations were estimated by using the experimental ultrasonic velocities and densities, enclosed in this paper. These values could be also evaluated by means the group contribution method proposed by Schaffs, 1963, when no experimental data are disposable [14]. The Free Length Theory (FLT) estimates the isentropic compressibility of a mixture attending to the free displacement of molecules as a main function of temperature. In the last few years different authors have compared the relative merits of the existent theories with FLT and CFT, both models resulting in relatively low deviations of computed isentropic compressibility from

experimental values for mixtures of different chemical nature. Figures $5 \mathrm{a}$ and $5 \mathrm{~b}$ gather deviations of isentropic compressibility as a function of $\mathrm{z}$ for extreme temperatures and standard condition. As observed larger deviations were computed for CFT at low $\mathrm{z}$ (pseudobinary compositions) at each temperature. The rest of the composition diagram shows positive deviations (the model determines lower values that experimental) around $10 \%$ of deviation. Estimations of FLT were poorer at every case; the model computes lower values than experimental (poorer results at low temperatures) offering similar values for the whole composition space (20-30\% of deviation). Attending to these facts, we arrive at the conclusion that the application of the
Collision Factor Theory predicts experimental data extremely well for all the studied mixtures, showing this procedure as an accuracy tool for isentropic compressibility data in these kind of systems, lower deviations being obtained at lower temperatures and compositions away from binary axis (bulk compositions).

Table 6. Root mean square deviations for estimated isentropic compressibilities for the ternary mixture at range 288.15-323.15 K.

\begin{tabular}{ccc}
\hline Temperature & $\begin{array}{c}\text { CFT } \\
\text { (eq. 25) }\end{array}$ & $\begin{array}{c}\text { FLT } \\
\text { (eq. 26) }\end{array}$ \\
\hline $23.15 \mathrm{~K}$ & 75.14 & 264.14 \\
$298.15 \mathrm{~K}$ & 53.92 & 441.61 \\
$288.15 \mathrm{~K}$ & 57.40 & 606.76 \\
\hline
\end{tabular}

(a)

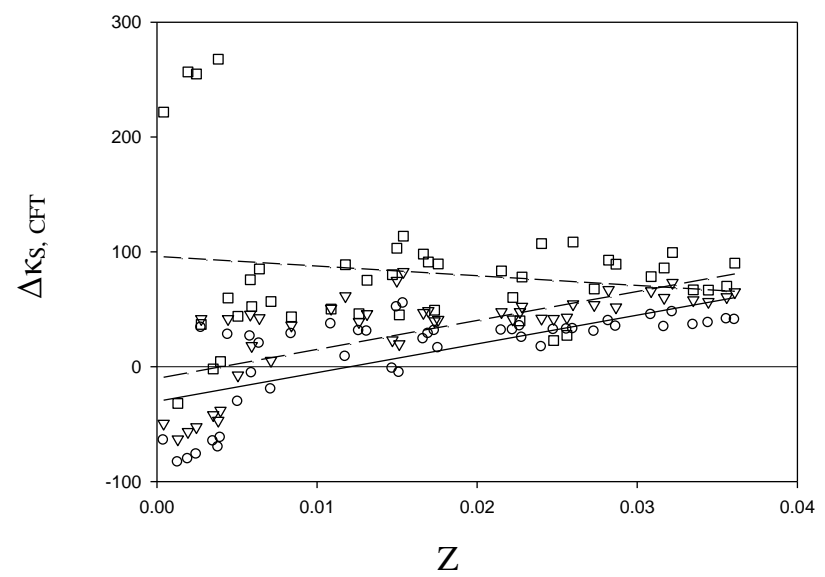

(b)

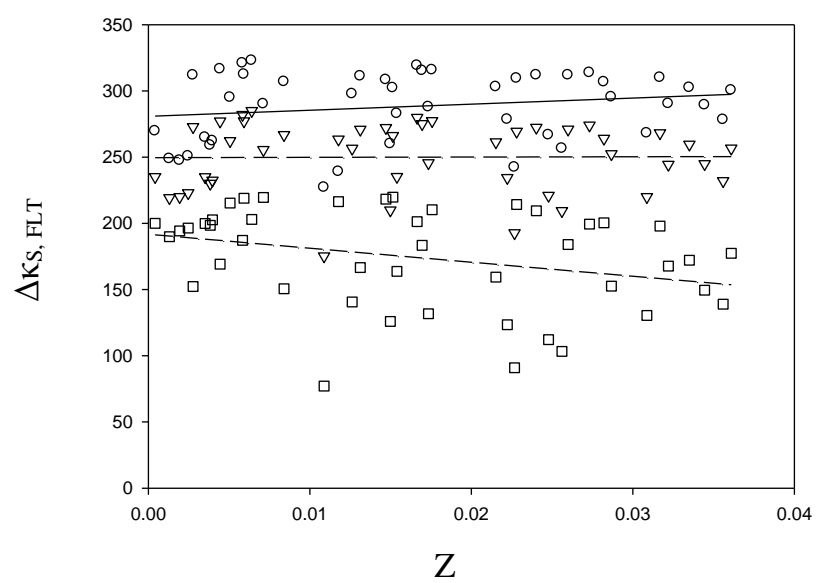

Figure 5. Deviations $\left(\triangle \kappa_{S}, T P a^{-1}\right)$ of the experimental measured values of isentropic compressibility at $(\mathrm{O}) 288.15$, (V) 298.15 and $(\square) 323.15 \mathrm{~K}$ from computed isentropic compressibility by (a) Collision Factor Theory (CFT) (eq. 25) and (b) Free Length Theory (FLT) (eq. 26). The average values at each temperature are shown through a right line (— for $288.15 \mathrm{~K}$, — - for $298.15 \mathrm{~K}$ and ------ for 323.15 $K)$.

\section{Discussion and Conclusions}

In the last few years, an increasing interest in density and ultrasonic applications has been noted. Nowadays, the measurements of both properties have been adequately used to study the nature of molecular systems and thermodynamic behavior in liquid mixtures. A survey of literature of binary 
and ternary liquid mixtures indicates that studies of density and ultrasonic velocity are useful in understanding the molecular interactions. The growing interest in the alcoholic multicomponent mixtures is caused by the occurrence in nature, as well as, their interest for different industrial applications. Ethanol has been used in the last few years as distillation entrainer or extractive solvent in the chemical industry, as a carrier or additive in food and pharmaceutical processes, and antimicrobial applications. As it occurs in heavy alcohols, this molecule contains an active hydrophilic hydroxyl group which is available to hydrogen-bond to analogous compounds, and a residual end conferring a variable degree of hydrophobicity on the molecule. The mixing properties, and behavior of ethanol vary with the nature of the chemical environment, operation conditions, and then, steric hindrance molecular effects as it was observed.

In this work, our attention was focused towards ternary mixtures of ethanol, water and one of the different flavor components in distilled alcoholic beverages due to its interest for food technology. In the mixture Ethanol+Water+2Propanol, the interaction of three solvents of polar nature is analyzed, the steric hindrance effect being of major importance. These factors are clearly demonstrated by the unusual packing characteristics of hydroxylic compounds in mixtures. The experimental values of the excess molar volumes and changes of isentropic compressibility provide information about ethanol + hydroxylic compounds interactions as the molar fraction of compounds is changed, as well as the packing efficiency. We present a study of the effect of temperature on these magnitudes in order to analyze the influence of the molecular chain residues, as well as, the progressive occultation, and relative position of hydroxyl groups towards hydrogen bonds formation, and mixing thermodynamics in Ethanol + Water + 2-Propanol. Apart from measuring density and ultrasonic velocity, other parameters such as isentropic compressibility, intermolecular free length, the van der Waals' constant, molecular radius, geometrical volume, molar surface area, available volume, volume at absolute zero, molar sound velocity, collision factor, specific acoustic impedance and Rao parameter of relative association, have also been evaluated for this ternary system. It is observed from the measured data that in the three components, an intense effect of temperature is produced (Table S1). In fact, in our data the curious effect of this magnitude on the ultrasonic velocity of water (inverse dependence of temperature with respect the other hydroxylic molecules) should be observed as shown in previous literature. The rigidity of the molecule becomes maximum at those composition points (low temperature and low 2-propanol composition) where velocity maxima occurs (Figure 1a). This may be interpreted due to the formation of strong hydrogen bonding, resulting into complex formation producing displacement of electrons and nuclei. The chemical interaction may involve the association due to hydrogen bonding, due to the dipole-dipole interaction or, to a lesser extension, due to the formation of charge-transfer complexes, all of them may lead to strong interaction. The occurrence of maximum velocity is for pure water at any temperature and then a subsequent decrease of velocity with further increase in concentration may be explained by an increment in entropy and a diminution of hydroxyl interaction power by steric hindrance of alcohol trace molecules. New molecules of ethanol and 2-propanol may be accommodated in the liquid matrix and this fact produces lower contraction when low concentration of water and ethanol are enclosed. The awakening of the intermolecular forces is probably the reason of the strong decrease in ultrasonic velocity. Further it may be seen from Fig. 1b where the change of isentropic compressibility is showed. As could be expected, attending to the molecular structure of solvents, three different trends of interaction should be observed: a hydrogen bond interaction domains the ethanol + water mixture yielding to intense contractive trend in the corresponding pseudo binary mixture (low 2-propanol composition). Second, an analogous trend is observed for water +2 -propanol, due to the similar functional groups enclosed (Figures $4 \mathrm{a}$ and $4 \mathrm{~b}$ ). And third, a slight progressive diminution of contraction occurs for equimolar compositions of alcohols when water component is eliminated (sigmoidal trend) (Figure $4 \mathrm{~b}$ ). The curious behavior is conditioned mainly by the steric hindrance over the aliphatic end of the heavy alcohol into the extremely ordered hydroxylic structure in such a way that diminution or increment of 2Propanol composition affects notably to the contractive tendency. This notorious effect was revealed previously $[8,9]$. The most non-ideal trend is provided for the lowest temperatures, so that higher values of isentropic compressibilities than ideal mixtures and then, a more rigid mixture. These results perfectly tallies with previous studies, showing as 2-Propanol is the key component for the contractive character of the mixture, moreover, high dilution or high concentration produces a diminution of the contractive trend. Temperature is a fact that in this case deals towards diminution of maxima of change of isentropic compressibility, probably due to an increasing difficult of accomodation of the aliphatic end of the heavy alcohol by molecular kinetics. In the last few years, different studies have pointed out the special "iceberg structure" of hydroxyl short molecules, specially aqueous mixtures of alcohols, and the intense modifications that this structure suffers as a function of composition and temperature. This special structure is specially sensitive to globular molecules with polar or slight polar groups (as 2-propanol) and inert solvents, which are difficulty enclosed. The propyl group ($\left.\mathrm{C}_{3} \mathrm{H}_{7}\right)$ is bulkier than ethyl group $\left(-\mathrm{C}_{2} \mathrm{H}_{5}\right)$ attached with hydroxyl group. Due to that, the short molecules in mixture experience greater steric hindrance as compared to the pure solvent situation or single interaction short solvent +2 Propanol. Steric hindrance awakens hydrogen bonding between oxygen and protons, resulting into formation of short mean life hydrogen bonds and less rigid mixtures at any specific temperature. The aqueous dilution of 2-Propanol shows contractive trend, due to the disruption of the polar interaction among water molecules by 2-Propanol diluent. This behavior is extrapolated to near ternary compositions such predominant interaction being attenuated by the composition increment of the third solvent (ethanol). The autoassociative behavior of ethanol is the cause of contractive tendency with water (polar hydroxyl group) and slight polar-dispersive interaction with the aliphatic end of 2Propanol. The mathematical structure of the Eq. 2 leads to analyze the ternary contribution (simultaneous interactions among all compounds into mixture) in what is referred to shape and sign (the two last terms of eq. 2). This contribution shows two pointing out questions: firstly, the considerable contribution to the derived property by ternary molecular interactions $(25 \%)$, although its a constant value for higher 
temperatures (in the range of this study); second, the different sign of this contribution attending to ethanol composition. Such trend is normal due to the evolution of the physical property as a function of molar fraction. The partial rupture of short alcohol + water close structure when the 2propanol is introduced is reflected in the isentropic compressibility by a strong and continuous variation, from negative values, towards increasing values until reach a maximum. The solvation layer around the ethanol is dissolved faster as temperature rises which may also be true for the whole set of the heavy alcohol functional family. A global weakening of intermolecular interactions is observed which could be used to account for the isentropic compressibilities. Both facts let us to understand the relative inaccuracy of the theoretical models in estimation these ternary values. Intermolecular free length shows an analogous behavior as reflected by isentropic compressibility for this mixture. The decreased compressibility brings the molecules to a closer packing resulting into a decrease of intermolecular free length. Intermolecular free length is a predominant factor in determining the variation of ultrasonic velocity in solutions. As the intermolecular free length decreases, ultrasonic velocity increases and vice-versa, showing a logical inverse behavior. The interdependence of intermolecular free length and the ultrasonic velocity has been evolved from a model for sound propagation proposed by Eyring and Kincaid [31]. The decrease in the values of isentropic compressibility and intermolecular free length with increase in ultrasonic velocity indicates that there is a significant interaction among molecules due to which structural arrangement is considerably affected. Figure $3 \mathrm{a}$ shows the non-ideal deviation in terms of $\mathrm{z}$ at $298.15 \mathrm{~K}$ being gathered an important deviation for low compositions (near of binary axis), in other words, at low compositions of ethanol, the molecules freely move between two physical interactions, a shorter length than ideally expected. In what is referred to the obtained values for the excess acoustic impedance at all concentrations of the mixtures, they are negative at any composition, decreasing their negative values as temperature diminishes. Acoustic impedance indicates how much sound pressure is produced by the vibration of molecules at a given frequency $(2 \mathrm{MHz})$ for a specific mixture as a function of temperature. Figure $3 b$ shows positive values at pseudobinary mixtures and negative values of $Z^{E}$ for medium and high values, this fact may be understood as an inverse sound pressure at low concentration that increases towards the centre of Gibbs triangle. This result is in line with those indicated above and coherent with previously published data on similar mixtures $[8,9]$.

Cubic equations of state were applied to predict the excess molar volumes of this ternary mixture. Both equations showed analogous merits in terms of correlation of open literature data (see Figure 4) using different mixing rules. As expected, rules 2 and 3 offer better capability of correlation with very similar deviations (see deviations into brackets for each binary mixture). The almost ideal interaction ethanol+2-propanol deals towards asymmetric contribution of the binaries, which in terms of symmetric cubic equations produces moderate deviations when ternary predictions are attempted (see the last row into Table 5). In what is referred to theoretical estimation for isentropic compressibility, as it could be observed in the Table 6 , the better deviations are showed for the CFT model, a slight overestimation being observed when the temperature is high. Both models are, at least, of qualitative accuracy to predict ternary values, showing the FLT a lower capacity for the whole composition range. Deviations yielded for the estimated ternary magnitude, also show temperature dependence, and can be considered as a satisfactory result, for supporting their validity as acceptable predictive tool, having in mind the high non-ideality of the studied ternary mixture, where high associative behavior phenomenon occurs and steric hindrance is a key factor for explaining molecular interaction.

\section{Acknowledgments}

S. Barros would like to thank FAPESB (Fundação de Amparo à Pesquisa do Estado da Bahia) for the financial support of a PhD. grant for developing this research.

\author{
Nomenclature \\ $\omega-$ acentric factor \\ $\rho$ - density $\left(\mathrm{g} / \mathrm{cm}^{3}\right)$ \\ $\delta \kappa_{\mathrm{S}}$ - changes of isentropic compressibility on mixing (TPa \\ 1) \\ $\rho_{\mathrm{c}}-$ critical density, $\mathrm{kgm}^{-3}$ \\ $\kappa_{\mathrm{S}}$ - isentropic compressibility, $\left(\mathrm{TPa}^{-1}\right)$ \\ $a_{i}, b_{i}, c_{i j}, d_{i j k}-$ derived properties correlation parameters \\ $b$ - van der Waals' constant $\left(\mathrm{cm}^{3} \mathrm{~mol}^{-1}\right)$ \\ $B$ - volume of molecule per mole, $\mathrm{m}^{3} \mathrm{~mol}^{-1}$ \\ $H^{\mathrm{E}}$ - excess molar enthalpy, $\mathrm{Jmol}^{-1}$ \\ $L_{f}-$ intermolecular free length $(\hat{A})$ \\ $M$ - molar mass $\left(\mathrm{gmol}^{-1}\right)$ \\ $n_{\text {DAT }}$ - number of experimental data \\ $P_{C}$ - critical pressure, $(\mathrm{K})$ \\ $Q$ - thermodynamic magnitude \\ $r$ - molecular radius, $(\mathrm{m}) \mathrm{R}_{\mathrm{A}}-$ \\ Rao parameter of relative association, $\left(\mathrm{m}^{3} \mathrm{~mol}^{-1}\right)\left(\mathrm{ms}^{-1}\right)^{1 / 3}$ \\ $R_{S}$ - molar sound velocity, $\left(\mathrm{ms}^{-1} \mathrm{~mol}^{-1}\right)$ \\ $S$ - collision factor \\ $T_{C}$ - critical temperature, $\mathrm{K}$ \\ $T_{\mathrm{R}}$ - reduced temperature, $\mathrm{K}$ \\ $u$ - ultrasonic velocity, $\mathrm{m} / \mathrm{s}$ \\ $V$ - mixing molar volume \\ $V_{0}$ - volume at absolute zero \\ $V^{\mathrm{E}}$ - excess molar volume $\left(\mathrm{cm}^{3} \mathrm{~mol}^{-1}\right)$ \\ $x_{i}$ - molar fraction \\ $Y$ - molar surface area, $\left(\mathrm{m}^{2} \mathrm{~mol}^{-1}\right)$ \\ $Z$ - specific acoustic impedance, $\left(\mathrm{kg} \mathrm{m}^{-2} \cdot \mathrm{s}^{-1}\right)$ \\ $\sigma-$ root mean square deviation
}

\section{Appendix}

Experimental data are disposable as supplementary information into Table ST1

\section{References:}

[1] M. Gaiser, G. M. Bell, A. W. Lim, N. A. Roberts, D. B. F. Faraday, R. A. Schultz, R. Grob, "Computer simulation of a continuous whisky still," J. Food Eng., 51, 27-31, 2002.

[2] C. A. Faúndez, J. O. Valderrama, V. H. Alvarez, "Phase equilibrium in binary aqueous mixtures of interest in 
alcoholic distillation using a modified PSRK equation of state," J. Phase Eq. and Diffusion, 25, 230-236, 2004.

[3] C. A. Faúndez, J. O. Valderrama, "Phase equilibrium modeling in binary mistures found in wine and must distillation,"J. Food Eng., 63, 577-583, 2004.

[4] O. Perez, L. Zumalacarregui, O. Gozá, “Simplificaciones en el cálculo de columnas de destilación alcohólica," Inf. Tecnol., 21, 103-112, 2010.

[5] J. Sacher, L. García-Llobodanin, F. López, H. Segura, J. R. Pérez-Correa, "Dynamic modeling and simulation of an alembic pear wine distillation," Food Bioprod. Process., 91, 447-456, 2013.

[6] C. A. Faúndez, G. A. Ramírez, J. O. Valderrama, "Thermodynamic consistency of low pressure equilibrium data of water+ congener mixtures using a versatile equation of state," J. Taiwan Inst. Chem. Eng., 68, 15-22, 2016.

[7] J. M. Resa, C. Gonzalez, J. M. Goenaga, M. Iglesias, "Density, refractive index, and speed of sound at 298.15 $\mathrm{k}$ and vapor-liquid equilibria at $101.3 \mathrm{kpa}$ for binary mixtures of ethyl acetate + 1-pentanol," J. Chem. Eng. Data, 49, 804-808, 2004a.

[8] J. M. Resa, C. Gonzalez, J. M. Goenaga, M. Iglesias, "Influence of temperature on the volumetric properties of ethanol + water + 1-pentanol," J. Serbian Chem. Soc., 69, 1073-1097, 2004b.

[9] J. M. Resa, C. Gonzalez, J. M. Goenaga, M. Iglesias, "Temperature dependence of excess molar volumes of ethanol + water + ethyl acetate," J. Sol. Chem. 33, 169198, 2004c.

[10] J. M. Goenaga, R. Gonzalez-Olmos, M. Iglesias J. M. Resa, "Measurement and modelling of phase equilibria for ethanol+water+1-pentanol at isobaric condition," Korean J. Chem. Eng., 23, 631-637, 2006.

[11] J. M. Resa, J. M. Goenaga, M. Iglesias, R. GonzalezOlmos, D. Pozuelo, "Liquid-liquid equilibrium diagrams of ethanol + water + (ethyl acetate or 1-pentanol) at several temperatures,",J. Chem. Eng. Data, 51, 13001305, 2006a.

[12] J. M. Resa, J. M. Goenaga, J. Lanz, M. Iglesias, "Vapor-liquid equilibrium of binary mixtures containing Ethyl acetate + 2-methyl-1-propanol and Ethyl acetate + 2-methyl-1-butanol at $101.3 \mathrm{kPa}$.,", J. Chem. Eng. Data, 51, 595-598, 2006b.

[13] M. Iglesias, B. Orge, M. Dominguez, J. Tojo, "Mixing properties of the binary mixtures of acetone, methanol, ethanol, and 2-butanone at 298.15 K," Phys. Chem. Liq., 37, 9-29, 1998.

[14] W. Schaaffs, Molekularakustic; eine Einfuehrung in die Zusammenhaenge zwischen ultraschall und molekuelstruktur in fluessigkeiten und gasen, ed. by Springer Verlag, Bonn, 1963.

[15] TRC Thermodynamic Tables, Thermodynamic Research Center, Texas A\&M University, College Station, TX, 1994.

[16] J. A. Riddick, W. B. Bunger, T. K. Sakano, Organic Solvents, Techniques of Chemistry, 4th ed., Vol. 2, ed. by Wiley-Interscience, New York, 1986.
[17] M. Iglesias, A. Torres, R. Gonzalez-Olmos, D. Salvatierra, "Effect of temperature on mixing thermodynamics of a new ionic liquid: 2-hydroxy ethylammonium formate (2-HEAF) + short hydroxylic solvents," J. Chem. Thermodyn., 40, 119-133, 2008.

[18] H. Iloukhani, B. Samiey, M. A. Moghaddasi, "Speeds of sound, isentropic compressibilities, viscosities and excess molar volumes of binary mixtures of methylcyclohexane +2 -alkanols or ethanol at T=298.15 K," J. Chem. Thermodyn., 38, 190-200, 2006.

[19] V. A. Del Grosso, C. W. Mader, "Speed of sound in pure water," J. Acous. Soc. Am., 52, 1442, 1972.

[20] C. Gonzalez, J. M. Resa, J. Lanz, M. Iglesias, "Intermolecular interactions in soybean oil + different organic solvents by ultrasonic velocity measurements," J. Food Eng., 77, 152-161, 2006.

[21] T. M. Aminabhavi, M. I. Aralaguppi, Sh. B. Harogoppad, R. H. Balundgri, "Densities, viscosities, refractive indices, and speeds of sound for methyl acetoacetate + aliphatic alcohols (C1-C8)," J. Chem. Eng. Data, 38, 31-39, 1993.

[22] M. Sovilj, "Densities of ternary liquid systems containing aliphatic alcohols at various temperatures," Bull. Chem. Technol. Macedonia, 18, 41-45, 1999.

[23] P. Navarro, M. Larriba, S. Garcia, J. Garcia, F. Rodriguez, "Physical properties of binary and ternary mixtures of 2-propanol, water, and 1-butyl-3methylimidazolium tetrafluoroborate ionic liquid," $J$. Chem. Eng. Data, 57, 1165-1173, 2012.

[24] A. Sikorska, N. Ponikwicki, A. Koniecko, B. B. Linde, "Comparative studies of the mixing effect on the thermal effusivity, compressibility, and molar volume for aqueous solutions of alcohols," Int. J. Thermophys., 31, 131-142, 2010.

[25] J. M. Goenaga, PhD Thesis, Facultad de Farmacia, Universidad del Pais Vasco, 2007.

[26] H. A. Zarei, F. Jalili, S. Assadi, "Temperature dependence of the volumetric properties of binary and ternary mixtures of water (1) + methanol (2) + ethanol (3) at ambient pressure (81.5 kPa)," J. Chem. Eng. Data, 52, 2517-2526, 2007.

[27] N. Radojkovic, A. Tasic, B. Grozdanic, M. Malic, "Excess volumes of acetone + benzene, acetone + cyclohexane, and acetone + benzene + cyclohexane at 298.15 K," J. Chem. Thermodyn., 9, 349-352, 1977.

[28] B. E. Poling, J. M. Prausnitz, J. P. O'Connell, The Properties of Gases and Liquids, 5th ed. McGraw-Hill, New York, 2001.

[29] G. Soave, "Equilibrium constants from a modified Redlich-Kwong equation of state," Chem Eng. Sci., 27, 1197-1203, 1972.

[30] D. Y. Peng, D. B. Robinson, "A new two-constant equation of state," Ind. Eng. Chem. Fundamentals, 15, 59-64, 1976.

[31] J. F. Kincaid, H. Eyring, "Free volumes and free angle ratios of molecules in miquids," J. Chem. Phys., 10, 620$629,1938$. 
[32] M. V. Kaulgud, "Ultrasonic velocity and compressibility in Binary liquid mixtures," Z. Phys. Chem., 36. 365-375, 1963.
[33] J. D. Pandey, S. N. Srivastav, “Temperature dependence of some acoustical and thermodynamical properties of letrafluoromethane and methane," Acoustica, 51, 66, 1982. 